\title{
Langerhans cell histiocytosis: a multisystem disorder
}

\author{
${ }^{1} \mathrm{~A}$ Munir, ${ }^{2} \mathrm{~N}$ Leech, ${ }^{3} \mathrm{KP}$ Windebank, ${ }^{4} \mathrm{~J}$ McLelland, ${ }^{5} \mathrm{GL}$ Jones, ${ }^{6} \mathrm{D}$ Mitra, ${ }^{7} \mathrm{~A}$ Jenkins, ${ }^{8} \mathrm{R}$ Quinton \\ ${ }^{1}$ Specialty Registrar Diabetes and Endocrinology; ${ }^{2}$ Consultant Diabetologist; ${ }^{3}$ Consultant Paediatric and Adolescent Oncology; ${ }^{4}$ Consultant \\ Dermatologist; ${ }^{5}$ Consultant Haematologist; ${ }^{6}$ Consultant Radiologist; ${ }^{7}$ Consultant Neurosurgeon; ${ }^{8}$ Consultant Endocrinologist, Royal Victoria \\ Infirmary, Newcastle upon Tyne, UK
}

\begin{abstract}
Langerhans cell histiocytosis can involve single or multiple organ/tissue systems and may go undiagnosed for years until it enters the clinician's differential diagnosis framework. We report on a young patient who initially presented with diabetes insipidus and subsequently with pyrexia of unknown origin. She progressed from single system Langerhans cell histiocytosis to multisystem involvement and remains in long-term remission following chemotherapy.
\end{abstract}

KEYWORDS Langerhans cell histiocytosis, diabetes insipidus

DECLARATION OF INTERESTS No conflicts of interest declared.

\author{
Correspondence to A Munir \\ Royal Victoria Infirmary, \\ Queen Victoria Road, \\ Newcastle upon Tyne \\ NEI 4LP, UK
}

tel. $+44(0) 7767235182$

e-mail atifl I3_2000@yahoo.co.uk

\begin{abstract}
A high index of suspicion is required to consider Langerhans cell histiocytosis ( $(\mathrm{LCH})$ as a possible unifying differential diagnosis in patients presenting with multisystem manifestations, which can lead to early diagnosis and hence better treatment outcomes. In this report we outline an index case to highlight the difficulties in diagnosis and recognition. Patients should be managed in centres with appropriate expertise and enrolled in ongoing clinical trials to provide more insight into the natural history of the disease and treatment outcomes.
\end{abstract}

\section{CASE REPORT}

A 24-year-old woman with type I diabetes mellitus presented with new-onset diabetes insipidus and was started on desmopressin. Anterior pituitary function was normal, but a magnetic resonance imaging (MRI) scan demonstrated pituitary stalk thickening. Although hypothalamic LCH was among the differential diagnoses at this point, the history of recurrent pneumothoraces (which would have enabled a near-definitive clinical diagnosis of multisystem LCH) was not appreciated. She subsequently failed to attend for planned endocrine treatment and imaging follow-up, presenting 18 months later with weight gain, amenorrhoea, confusion, memory impairment, pyrexia of unknown origin and deranged liver function tests. There was also a history of vulval irritation, for which she had been self-medicating with antifungals, along with rapidly evolving pruritic, nongenital skin lesions.

Endocrine evaluation confirmed panhypopituitarism and severe vitamin $D$ deficiency. Abdominal imaging revealed multiple focal deposits in her liver that appeared infective or neoplastic, but on liver biopsy appearances were more consistent with cholangitis and immuno- histochemistry was inconclusive. A repeat pituitary MRI scan showed an inflammatory hypothalamic mass (that subsequently showed hyperintense uptake on FI8 fluorodeoxyglucose positron emission tomography/ computed tomography [I8-FDG PET-CT]) with a normal appearing pituitary gland. A stereotactic biopsy of this mass showed $\mathrm{SI} 00$ proteins and cluster of differentiation (CD la) immunopositive cells, establishing a diagnosis of $\mathrm{LCH}$. Further investigations were arranged to determine if this was the unifying diagnosis for her other systemic manifestations and to evaluate the extent of the disease. A biopsy of her skin rash also showed abundant histiocytes that also stained positive for CDIa and SI00 and a high-resolution chest $\mathrm{CT}$ scan revealed multiple cysts and nodules bilaterally, typical of $\mathrm{LCH}$, although bone marrow examination did not show any involvement.

With a proven diagnosis of multisystem $\mathrm{LCH}$ and initiation of appropriate endocrine replacement therapy, the focus shifted to disease-specific therapy. Although neurocognitive assessment was influenced by a strong element of anxiety, there was a definite defect of shortterm memory and information-retention. We were concerned that caudal spread of the neuroinflammatory process towards the mamillary bodies might result in an irreversible Korsakoff-like syndrome that could prove damaging to her prospects of independent living, particularly in the context of poorly-controlled type I diabetes. However, her insulin requirements indicated significant obesity-related insulin resistance, making us reluctant to initiate conventional therapy with high-dose steroids, with the risk of inducing major destabilisation of glycaemic control.

There were no published guidelines at the time of diagnosis and her treatment decisions were taken in view of the evidence available. She failed to respond to a 
therapeutic trial of the protein kinase inhibitor imatinib, but experienced significant clinical and radiologic improvement over the course of eight cycles of the antimetabolite agent cladribine. Systemic symptoms, including pyrexia, resolved and neurocognitive function improved, with subsequent MRI scans showing major reduction in the size of the inflammatory hypothalamic mass after just two cycles of cladribine, which reached a plateau with subsequent courses. However, her skin lesions remained problematic, but fortunately responded to periodic pamidronate infusions and, overall, her disease remains in remission. A recent I8-FDG PET CT scan did not show any abnormal uptake.

\section{DISCUSSION}

Histiocytosis encompasses a group of diverse idiopathic disorders which have a common primary trigger: accumulation of monocytes, macrophages and dendritic cells in the affected tissues. Viral infections, cellular and immune dysfunction, neoplastic mechanisms, genetic factors and their combinations have been implicated in aetiology and pathogenesis. Estimated prevalence is one to two cases of LCH per million population. It can affect all age groups, ranging from neonates to adults and can be local or systemic. ${ }^{1-7}$ Patients may be asymptomatic. Presenting symptoms or signs from published studies in order of decreasing frequency are: skin rash, dyspnoea, polydipsia and polyuria, bone pain, lymphadenopathy, weight loss, fever, gingival hypertrophy, ataxia and memory problems.

Dermatological manifestations include brown to purplish papules. Cystic or nodular lesions within the lung can lead to the destruction of parenchymal tissue, so a spontaneous pneumothorax is often the first sign of $\mathrm{LCH}$. Endocrine manifestations include diabetes insipidus, anterior hypopituitarism and a goitre. About $80 \%$ of patients presenting with diabetes insipidus develop a permanent deficiency of anterior pituitary hormones. ${ }^{8,9}$ Primary sites of bone involvement in order of frequency include the jaw, skull, vertebrae, pelvis, extremities and ribs. Central nervous system (CNS) involvement results in inflammation, followed by neurodegeneration. Splenic involvement can cause splenomegaly. Hepatic manifestations include hepatomegaly and tumour-like or cystic lesions which can be accompanied by elevated liver enzymes, hypoalbuminaemia or clotting factor deficiencies. Sclerosing cholangitis is a recognised serious complication, with liver biopsy showing lymphocytic infiltration of the biliary tree, though classic immunopositive histiocytes are rarely found. ${ }^{10,11}$ Bone marrow involvement is uncommon in adults, but can be associated with anaemia or leukopenia.
TABLE | Clinical classification of Langerhans cell histiocytosis

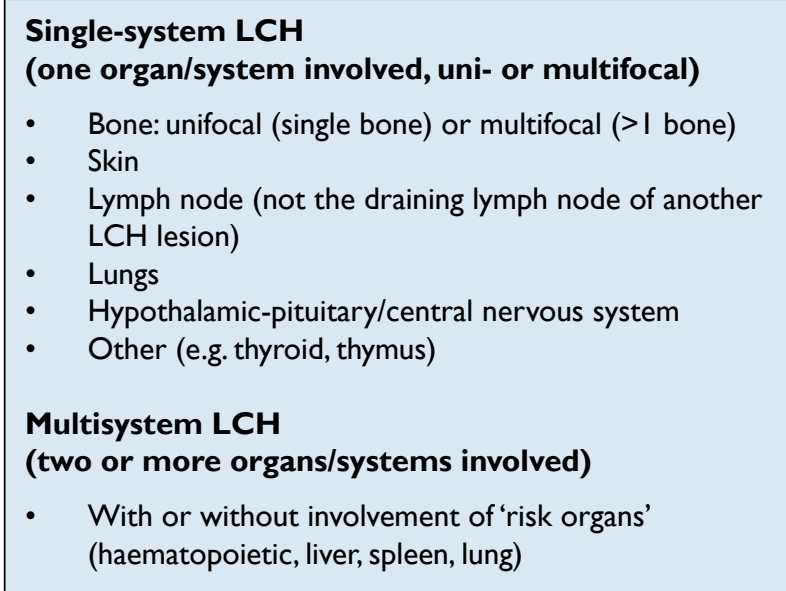

The length of time from the first symptom(s) to diagnosis can be very long. Diabetes insipidus is frequently the initial symptom, sometimes starting in childhood. Langerhans cell histiocytosis may remain unrecognised until other systemic symptoms occur later in life. Except for pulmonary $\mathrm{LCH}$, which can be diagnosed on high resolution computed tomography (HRCT) imaging characteristics alone, the diagnosis is based on histological and immunophenotypic examination of lesional tissue (biopsy of CNS tissue or skin lesion, or bone marrow exam), with core features being the morphologic identification of the characteristic LCH cells. Positive staining with CDIa and/or langerin (CD207) is required for definitive diagnosis. ${ }^{12}$ A PET scan is considered to be the most sensitive functional test to detect LCH lesions and can be used to evaluate response to therapy.

Clinical classification is based on the extent of involvement (Table I). Optimal treatment has not been established. Single-system disease may warrant monitoring or local therapy. ${ }^{13}$ Significant disease variation and spontaneous regression in up to $20 \%$ of patients complicates comparisons of current therapies. Several agents including chemotherapy have been effective in treatment. According to Histiocytosis Society guidance (2009) a combination of prednisolone and vinblastine has been proven to be effective and is therefore the standard initial therapy for all patients who should receive systemic therapy (Table 2). ${ }^{14-16}$ Long-term survival

TABLE 2 Indications for systemic therapy in Langerhans cell histiocytosis

- $\quad$ Single-system LCH with 'central nervous system risk' lesions (craniofacial lesions)

- $\quad$ Single-system LCH with multifocal bone lesions

- $\quad$ Single-system LCH with 'special site' lesions (odontoid peg or vertebral lesions with soft tissue extension)

- Multisystem LCH with/without involvement of 'risk organs' (haematopoietic, liver, spleen, lung) 
is best for those without the involvement of high risk organs (haematopoietic, liver, spleen, lung) and who respond to standard initial therapy.

Evidence to date is insufficient to support an optimal course of treatment for patients with progressive multisystem LCH who fail to respond to standard therapy. Results for patients treated with a combined regimen of 2-chlorodeoxyadenosine (2-CdA, cladribin, leustatin) and cytarabine (ara-C), as well as stem cell transplantation after undergoing a reduced intensity conditioning regimen are promising. ${ }^{17,18}$ All patients should be enrolled in clinical trials organised by the Histiocyte Society and should have long-term follow-up with a multi-disciplinary team with knowledge of $\mathrm{LCH}$.

\section{CONCLUSION}

Langerhans cell histiocytosis in adults has many (but not all) of the characteristics of neoplasia and can be considered an 'orphan disease' as patients may present with single-system involvement to any specialty and may remain undiagnosed. Even when accurately diagnosed, no organised treatment protocols are in place to define optimal treatment. Diagnosis is easier when advanced multisystem involvement is observed, but a high index of suspicion is required to establish a diagnosis of $\mathrm{LCH}$ when the disease is focal. A thorough workup is essential to determine the extent of the disease and possible complications. Patients need long-term follow-up for early detection of recurrence and consideration of treatment.

\section{REFERENCES}

I Kannourakis G,Abbas A.The role of cytokines in the pathogenesis of Langerhans cell histiocytosis. Br J Cancer Suppl 1994; 23:S37-40.

2 Egeler RM, Favara BE, Van Meurs $M$ et al. Differential in situ cytokine profiles of Langerhans-like cells and T cells in Langerhans cell histiocytosis: abundant expression of cytokines relevant to disease and treatment. Blood 1999; 94:4195-201.

3 Ornvold K, Carstensen H, Larsen JK et al. Flow cytometric DNA analysis of lesions from 18 children with langerhans cell histiocytosis (histiocytosis x). Am J Pathol 1990; 136:1301-7.

4 Halton J,Whitton A,Wiernikowski J et al. Disseminated Langerhans cell histiocytosis in identical twins unresponsive to recombinant human alpha-interferon and total body irradiation. Am J Pediatr Hematol Oncol I 992; 1 4:269-72.http://dx.doi.org/ I0.1097/00043426199208000-00016

5 de Graaf JH,Tamminga RY, Kamps WA et al. Expression of cellular adhesion molecules in Langerhans cell histiocytosis and normal Langerhans cells. Am J Pathol 1995; |47:1 |61-71.

6 Baumgartner I, von Hochstetter A, Baumert B et al. Langerhans cell histiocytosis in adults. Med Pediatr Oncol 1997; 28:9-14. http:// dx.doi.org/I0.1002/(SICI) I096-9IIX(I9970I)28: I<9::AID MPO3>3.0.CO;2-P

7 Malpas JS. Langerhans cell histiocytosis in adults. Hematol Oncol Clin North Am 1998; 12:259-68. http://dx.doi.org/10.1016/S0889. 8588(05)70509-8

8 García Gallo MS, Martínez MP, Abalovich MS et al. Endocrine manifestations of Langerhans cell histiocytosis diagnosed in adults. Pituitary 2010; 13:298-303. http://dx.doi.org/I0.1007/s I I I02-0 I00233-8

9 Kaltsas GA, Powles TB, Evanson J et al. Hypothalamo-pituitary abnormalities in adult patients with langerhans cell histiocytosis: clinical, endocrinological, and radiological features and response to treatment. J Clin Endocrinol Metab 2000; 85:1370-6. http://dx.doi. org/10.1210/jc.85.4.1370

I0 Kaplan KJ, Goodman ZD, Ishak KG. Liver involvement in Langerhans' cell histiocytosis: a study of nine cases. Mod Pathol 1999; 12:370-8.

II Haas S, Theuerkauf I, Kühnen A et al. [Langerhans' cell histiocytosis of the liver. Differential diagnosis of a rare chronic destructive sclerosing cholangitis.] Pathologe 2003; 24:1 19-23. German.

12 Lau SK, Chu PG, Weiss LM. Immunohistochemical expression of langerin in Langerhans cell histiocytosis and non-Langerhans cell histiocytic disorders. Am J Surg Pathol 2008; 32:6I5-9. http://dx.doi. org/10.1097/PAS.0b013e31815b212b

I3 Broadbent V, Gadner H. Current therapy for Langerhans cell histiocytosis. Hematol Oncol Clin North Am 1998; I2:327-38. http:// dx.doi.org/I0.1016/S0889-8588(05)705I3-X

14 Gadner H, Heitger A, Grois $N$ et al. Treatment strategy for disseminated Langerhans cell histiocytosis. DAL HX-83 Study Group.Med Pediatr Oncol 1994;23:72-80.http://dx.doi.org// 0.1002/ mpo.2950230203

15 Gadner H, Grois N, Potschger $U$ et al. Improved outcome in multisystem Langerhans cell histiocytosis is associated with therapy intensification. Blood 2008; I I I:2556-62. http://dx.doi. org/I0.I I82/blood-2007-08-1062 I I

16 McClain KL. Drug therapy for the treatment of Langerhans cell histiocytosis. Expert Opin Pharmacother 2005; 6:2435-4l. http:// dx.doi.org/I0.1517//4656566.6.14.2435

17 Bernard F, Thomas C, BertrandY et al. Multi-centre pilot study of 2-chlorodeoxyadenosine and cytosine arabinoside combined chemotherapy in refractory Langerhans cell histiocytosis with haematological dysfunction. Eur J Cancer 2005; 4I:2682-9. http:// dx.doi.org/10.1016/j.ejca.2005.02.007

18 Steiner M, Matthes-Martin S, Attarbaschi A et al. Improved outcome of treatment-resistant high-risk Langerhans cell histiocytosis after allogeneic stem cell transplantation with reduced-intensity conditioning. Bone Marrow Transplant 2005; 36:2 I5-25. http://dx.doi.org/I0.1038/sj.bmt.I7050I5 